\title{
Epigenetic epidemiology as a tool to understand the role of immunity in chronic disease
}

\author{
"Knowing that the components of the immune system are \\ epigenetically unique, it is possible to apply this knowledge to \\ the epigenome-wide association studies, so that 'true' signals are \\ uncovered that are independent of shifts in immunity."
}

First draft submitted: 9 May 2016; Accepted for publication: 17 May 2016; Published online: 13 July 2016

Keywords: DNA methylation • epidemiology • epigenetics • immunology

The field of 'translational science' was described by then NIH Director Zerhouni in 2005 as "an emerging discipline that encompasses both the acquisition of new knowledge about health and disease prevention, preemption, and treatment and the methodologic research necessary to develop or improve research tools" [1]. Epigenetics as a field has spanned the scientific landscape, including intensive in vitro investigations into the fundamentals of epigenetic regulation as well as numerous large epidemiologic and clinical studies probing the links between epigenetic marks and human health. Unfortunately, there has been little cross-talk between these very focused investigators. Here, we would like to highlight one area that stands poised for collaborative investigation, as it is now clear that a translational approach is required to coherently move the science forward.

The fields of epigenetics, immunology and epidemiology are rapidly converging; epidemiologists are widely applying the new tools of epigenetics to study DNA methylation in blood. This approach has the appeal of directly addressing the 'developmental origins of adult disease' hypothesis put forth by Barker [2] by assessing potentially environmentally responsive epigenetic setpoints that may be critical in later disease sensitivity. Primarily these epigenome-wide association studies (EWAS) have been conducted in blood. This is done for the obvious reason that it is the tissue most easily sampled and available in large epidemiologic studies. Herein lies the important connections between epidemiology, epigenetics and immunology as bloodbased measures of epigenetics are inherently interrogating the immune system, which is a tissue comprised of numerous subtypes of cells that vary by lineage.

For many years it has been recognized that each tissue has a unique epigenome. This was perhaps first definitively accepted for Treg cells were FOXP3 is uniquely demethylated in a differentially methylated region (DMR) [3]. Subsequently, using the first generation of DNA methylation arrays (which were small and biased towards cancer genes), we demonstrated that DNA methylation signatures could be identified that differentiates normal tissues according to cell lineage [4]. Since that time sophisticated experiments have established that remodeling of the epigenome is an essential element of hematopoiesis, and this remodeling process leads to progressively restricted immune subtypes [5]. The very recent publication by Orlanski et al. [6], elegantly demonstrates that TET-mediated demethylation at B-cell enhancer elements is the gatekeeping event for B-cell maturation. By extension one can assume that this is a generalized mechanism for immune cell differentiation, and that highly specialized immune cells may also develop through a similar mechanism of targeted demethylation. It is now clear that DNA methylation provides a chemically stable mark for cell fate decisions.
Epigenomics
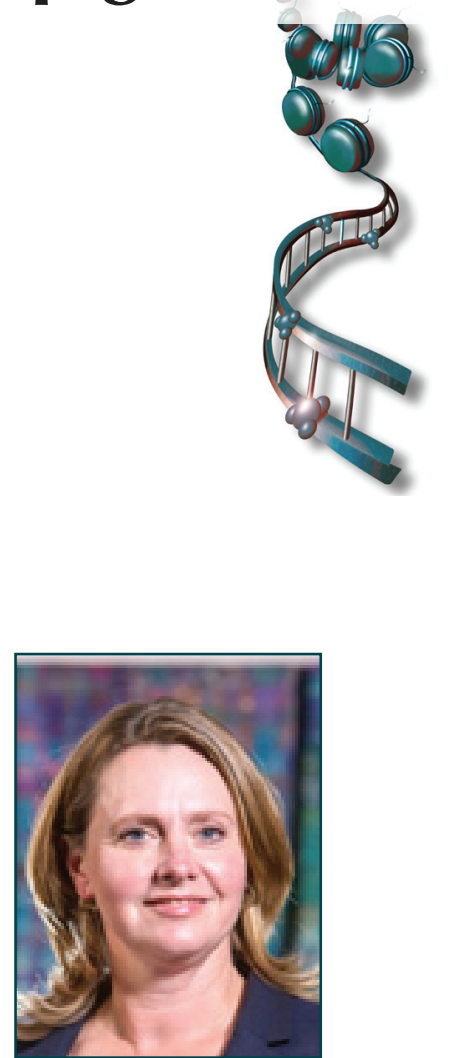

Heather H Nelson

Department of Prevention \& Etiology, Masonic Cancer Center, University of Minnesota, Minneapolis, MN 55455, USA

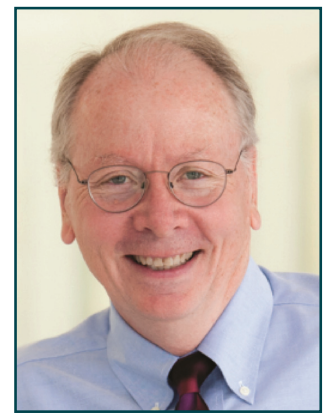

Karl T Kelsey

Author for correspondence: Departments of Epidemiology, Laboratory Medicine \& Pathology,

Brown University,

Providence, RI 02912, USA Karl_Kelsey@Brown.edu
Future 
Our recent work resonates with this, as we began to explore the extent to which stable and heritable changes in DNA methylation are produced during immune activation within cells of a common lineage identity [7]. Transcriptomic studies previously implicated genome wide alterations in gene expression during pathogen- and cytokine-induced immune activation in different cell types [8-12], but our study began to define the role of DNA methylation in natural killer (NK) activation [7]. In these experiments we demonstrated that NK cell activation led predominately to CpG hypomethylation (81\% of significant loci). Bioinformatics analysis confirmed that NK activation was associated with methylation changes at non-coding and gene-associated differentially methylated sites (DMS) that are enriched for immune related functions (i.e., immune cell activation). The DMR signature of activated NKs revealed similarities with T-cell activation, but also provided unique biomarker candidates of NK activation.

\section{"Agnostic interrogation of the immune DNA methylation footprint, with knowledge of most of the known immune subtype methylation profiles, would allow for the identification of novel immune states in disease."}

Knowing that the components of the immune system are epigenetically unique, it is possible to apply this knowledge to the EWAS, so that 'true' signals are uncovered that are independent of shifts in immunity. This approach was first devised by Houseman et al. [13] who developed a method for deconvolution of whole blood leukocyte DNA methylation signatures, allowing for quite precise estimation of major cell subtype prevalence from methylation array data (more recently updated in [14]). The method takes advantage of the unique $\mathrm{CpG}$ loci associated with individual cell subtypes, defining differentially methylated regions (DMRs). This methodology has been extensively applied by epidemiologists and there has been an explosion of recent epigenetic epidemiology investigations that use EWAS to identify epigenetic events that segregate with either environment exposures or disease. The tenor of these investigations has been to identify epigenetic variation that occurs independent of immune cell modulation. With this approach epidemiologists can address the aforementioned 'developmental origins of adult disease' hypothesis. However, what our most recent paper on NK activation illustrates is that it is entirely possible that the results of the EWAS are signals from immune subtypes whose DNA methylation profile is not yet represented in the currently defined 'libraries' (e.g. other activated cell types or other rare cell subtypes) or are not easily defined by simple flow cytometry experiments (e.g., myeloid derived suppressor cells).

There are now several examples of the problems inherent in interpretation of the EWAS. Bauer et al. [15] have very nicely demonstrated how more precise and specific immunological data are needed to properly interpret the results of EWAS. They focused on the CpG site cg19859270, which is located in the gene body of GPR15, an orphan chemoattractant receptor and co-receptor for HIV. This locus is consistently demethylated in blood samples from smokers [16] and this has been interpreted as a direct effect of tobacco smoke on leukocytes. However, the Bauer et al. data show that the observed decreased methylation at this site in DNA derived from circulated white blood cells is highly correlated with the proportion of $\mathrm{CD}^{+} \mathrm{GPR} 15^{+}$ expressing $\mathrm{T}$ cells. Hence, smoking (which is an irritant and immunoactive compound) changes the relative abundance of this T-cell subset and, as a result, the prevalence of methylation at this site is altered. It is most likely that the smoking-EWAS have identified not a smoking-sensitive gene/CpG, but rather a change in T-cell subsets. Similarly, our data has shown that activation of NK cells is also associated with changes in several CpG loci previously associated with smoking, again strongly suggesting that this exposure alters the underlying leukocyte profile rather than 'demethylating' specific $\mathrm{CpG}$ sites [7]. As a final example, consider recent data from Pacis et al. [17], demonstrating that infection of human dendritic cells (DCs) with a live pathogenic bacteria is associated with rapid and active demethylation at thousands of loci, independent of cell division. It is not difficult to imagine how this might complicate any EWAS of disease associated with an infectious process.

This knowledge presents a perplexing problem on how to best approach the role of immunity in chronic disease. First, it is crucial for the population scientists to recognize that the current approaches are incomplete and thus potentially flawed. Going forward, it would be extremely helpful to carefully isolate specific, highly specialized cell populations and identify their unique epigenetic footprint. This approach was successful for mapping DMRs associated with NK activation [7] and has also proved useful in understanding B cell development [6]. Indeed, the 'Blueprint' consortia is making available sequence data for a large number of subtypes of immune cells and these may be critical in creating a truly universal library for epidemiologic study of multiple populations [18]. However, it is unlikely that all possible subtypes of relevance for chronic disease can be isolated a priori and epigenetically mapped. We posit that results from epidemiology studies could be equally informative to the field of immunology in 
identifying novel cell types important in the development of disease. Agnostic interrogation of the immune DNA methylation footprint, with knowledge of most of the known immune subtype methylation profiles, would allow for the identification of novel immune states in disease. This could prove extremely useful for discovery in basic immunology.

The future for epigenetics research clearly is collaborative; it includes identification of these cells, understanding their relationship to disease, and identifying how to harness that information for treatment. There is now an urgent need for the disciplines of epigenetics, epidemiology and immunology to come together to interpret the role of epigenetics in environmental

\section{References}

1 Zerhouni EA. Translational and clinical science - time for a new vision. N. Engl. J. Med. 353(15), 1621-1623 (2005).

2 Barker DJ. The fetal and infant origins of adult disease. BMJ 301(6761), 1111 (1990).

3 Baron U, Floess S, Wieczorek G et al. DNA demethylation in the human FOXP3 locus discriminates regulatory $\mathrm{T}$ cells from activated FOXP3 ${ }^{+}$conventional T cells. Eur. J. Immunol. 37(9), 2378-2389 (2007).

4 Christensen BC, Houseman EA, Marsit CJ et al. Aging and environmental exposures alter tissue-specific DNA methylation dependent upon CpG island context. PLoS Genet. 5(8), e1000602 (2009).

5 Natoli G. Maintaining cell identity through global control of genomic organization. Immunity 33(1), 12-24 (2010).

6 Orlanski S, Labi V, Reizel Y et al. Tissue-specific DNA demethylation is required for proper $\mathrm{B}$-cell differentiation and function. Proc. Natl Acad. Sci. USA 113(18), 5018-5023 (2016).

7 Wiencke JK, Butler R, Hsuang G et al. The DNA methylation profile of activated human natural killer cells. Epigenetics 11(5), 363-380 (2016).

8 Best JA, Blair DA, Knell J et al. Transcriptional insights into the CD8 ${ }^{+} \mathrm{T}$ cell response to infection and memory T-cell formation. Nat. Immunol. 14(4), 404-412 (2013).

9 Bezman NA, Kim CC, Sun JC et al. Molecular definition of the identity and activation of natural killer cells. Nat. Immunol. 13(10), 1000-1009 (2012).

10 Dybkaer K, Iqbal J, Zhou G et al. Genome wide transcriptional analysis of resting and IL2 activated human exposure and chronic disease. Here, we call for a coordinated collaboration of immunologists and populations scientists, to refine study designs and to interpret the resulting data in a biologically meaningful way.

\section{Financial \& competing interests disclosure}

The authors' work was supported in part by funding from the NIH (grants R01CA207110 and R35CA197292). The authors have no other relevant affiliations or financial involvement with any organization or entity with a financial interest in or financial conflict with the subject matter or materials discussed in the manuscript apart from those disclosed.

No writing assistance was utilized in the production of this manuscript.

natural killer cells: gene expression signatures indicative of novel molecular signaling pathways. BMC Genomics 8, 230 (2007).

$11 \mathrm{Hu}$ G, Chen J. A genome-wide regulatory network identifies key transcription factors for memory CD8 ${ }^{+} \mathrm{T}$-cell development. Nat. Commun. 4, 2830 (2013).

12 Ma D, Cao W, Kapur A et al. Differential expression of proteins in naive and IL-2 stimulated primary human NK cells identified by global proteomic analysis. J. Proteomics 91, 151-163 (2013)

13 Houseman EA, Accomando WP, Koestler DC et al. DNA methylation arrays as surrogate measures of cell mixture distribution. BMC Bioinformatics 13, 86 (2012).

14 Koestler DC, Jones MJ, Usset J et al. Improving cell mixture deconvolution by identifying optimal DNA methylation libraries (IDOL). BMC Bioinformatics 17, 120 (2016).

15 Bauer M, Linsel G, Fink B et al. A varying T cell subtype explains apparent tobacco smoking induced single $\mathrm{CpG}$ hypomethylation in whole blood. Clin. Epigenetics 7(1), 81 (2015).

16 Gao X, Jia M, Zhang Y, Breitling LP, Brenner H. DNA methylation changes of whole blood cells in response to active smoking exposure in adults: a systematic review of DNA methylation studies. Clin. Epigenetics 7, 113 (2015).

17 Pacis A, Tailleux L, Morin AM et al. Bacterial infection remodels the DNA methylation landscape of human dendritic cells. Genome Res. 25(12), 1801-1811 (2015).

18 Martens JH, Stunnenberg HG. BLUEPRINT: mapping human blood cell epigenomes. Haematologica 98(10), 1487-1489 (2013). 